


\title{
Role of Retrograde Trans-Popliteal Access for Surgical High-Risk Patients with TASC II-D Femoro-Popliteal Occlusive Disease: A Single Center Experience
}

\author{
Mohamed Mahmoud Zaki, Amr Nabil Kamel, Amr Abdelghaffar Hanfy Mahmoud \\ Department of Vascular Surgery, Faculty of Medicine, Ain Shams University, Egypt
}

Objectives: To compare the safety and efficacy of retrograde trans-popliteal access in total Superficial Femoral Artery (SFA) occlusions (TASK II-D) in surgical high-risk patients, in the presence of a patent popliteal artery, after unsuccessful antegrade lesion crossing attempts.

Patients and methods: This is a retrospective study including 58 surgical high-risk patients with TASC II D femoro-popliteal occlusive diseases. After failure of antegrade access to cross the occlusion in 9 patients, medial retrograde trans-popliteal access was successfully achieved, followed by snaring of the retrograde wire through rendezvous technique. Perioperative complications and technical success were compared between different access groups.

Results: 58 patients were included in the study; 15 men (25.9\%) and 43 women (74.1\%). 42 patients (72.4\%) underwent contralateral retrograde access at Common Femoral Artery (CFA), 7 patients $(12.1 \%)$ ipsilateral antegrade access at CFA, 9 patients (15.5\%) ipsilateral retrograde access at popliteal artery. All patients were ASA grade 4. Technical success was highest with contralateral retrograde entailing 40 cases $(95.2 \%)$, followed by ipsilateral ante-grade access ( 6 cases $-85.7 \%$ ) and lowest with ipsilateral retrograde (7 cases- $77.8 \%$ ).

Conclusion: Transpopliteal retrograde approach can be utilized safely and efficiently using a medial infracondylar retrograde popliteal puncture, with the patient in the supine position. Furthermore, this retrograde popliteal approach can be considered a valid alternative for SFA or proximal PA recanalization after a failed antegrade approach, especially in high risk surgical patients (ASA 4).

Keywords: Endovascular management of femoral artery complex total occlusion, TASC II D Femoro-popliteal occlusive Disease, Retrograde popliteal access, Trans-popliteal access.

Conflicts of interest: There are no conflicts of interest or sources of funding that any of the authors receive to disclose.

Type of Research: Single center, non-randomized Cohort study.

Key Findings: 58 TASC II D femoropopliteal angioplasty procedures were done. Technical success was highest with contralateral retrograde (40 cases - 95.2\%), followed by ipsilateral ante-grade access ( 6 cases $-85.7 \%$ ) and lowest with ipsilateral retrograde (7 cases-77.8\%).

Take home message: Retrograde popliteal approach can be considered a safe, effective, and valid alternative for Superficial Femoral Artery, or proximal popliteal recanalization after a failed antegrade approach.

\section{Summary}

Retrograde approach showed comparable technical success and no perioperative complications in this retrospective assessment of 58 patients with femoropopliteal occlusions. The authors conclude that retrograde approach is a safe and reliable alternative to contralateral retrograde or ipsilateral antegrade access after failure of TASK IID femoropopliteal occlusion antegrade crossing attempts.

\section{Introduction}

Peripheral arterial disease (PAD) is usually caused by atherosclerosis that leads to stenosis (narrowing) or blockage in the major vessels supplying the lower extremities. Approximately $10 \%$ of the worldwide population have PAD. ${ }^{1}$ The vast majority of people with PAD are either asymptomatic or do not have any functional limitation. ${ }^{2}$ Many people with PAD, 
however, have limited walking ability and therefore reduced quality of life. Besides the limited walking ability, people with advanced PAD/critical limb ischemia are at risk of limb loss. In addition to affecting the limbs, PAD is a "manifestation of systemic atherosclerosis that involves other major circulation, such as the cerebral and coronary arteries". ${ }^{3}$ Patients diagnosed with PAD are at a twoto three-fold increased risk of mortality, myocardial infarction (MI) and stroke compared to age- and sex-matched population without PAD. ${ }^{4}$

Endovascular treatment is an attractive alternative to open surgical procedures for peripheral arterial disease (PAD) due to the less associated periprocedural risk. Various therapeutic strategies can be planned to treat long femoro-popliteal (FP) lesions, such as self-expandable stent, drug eluting balloon or stent, or covered stent. ${ }^{5}$

BASIL trial reported that up to 2 years, there was no statistically significant difference in overall survival or amputation-free survival by intention-to-treat assignment between surgery and angioplasty in severe limb ischemia. ${ }^{6}$ However, post hoc analysis demonstrated that beyond 2 years, patients initially randomized to open bypass surgery had superior clinical outcomes. ${ }^{7}$ Consequently, in patients who are not at high risk for surgery, bypass surgery is indicated for long (i.e. >_25 cm) SFA lesions when an autologous vein is available and life expectancy is $>2$ years. $^{7}$

Endovascular treatment for chronic total occlusion of the femoro-popliteal artery is usually managed from an antegrade ipsilateral common femoral artery approach, or via a retrograde contralateral approach in absence of a sufficient working distance in the SFA. Manipulation and pushability through the retrograde contralateral approach may be very challenging, especially in patients with high iliac tortuosity and previous aorto-bi-iliac bypass for aorto-iliac occlusive or aneurysmal disease. Moreover, retrograde contralateral access may be difficult or contraindicated in flush occlusions of the SFA without a proximal stump, obesity, surgical scars in the groin, and lesions involving CFA.

In these situations, when antegrade access fails, retrograde trans-popliteal approach may be an efficient alternative, especially in high risk surgical patients. A medial supra or infra-genicular approach to popliteal artery access was first described by Tonnessen and his colleagues in 1988; however, an antero-lateral approach has been recently gaining popularity with overcoming the obstacle of post-intervention puncture site sealing through initial sheathless access till rendezvous, sphygmomanometer cuff inflation, low pressure ballooning and fibrin blood injection at the access site. 9.10

\section{Objective}

This is a retrospective analysis of data collected from patients with high risk for surgery (ASA 4), and TASK II D femoro-popliteal occlusive disease. This study aims at detecting the safety and efficacy of retrograde popliteal access after failure of antegrade crossing of total SFA arterial occlusions in endovascular management for surgical highrisk patients with TASC II D femoro-popliteal occlusive disease. Primary end point is limb salvage, secondary end points include technical success and perioperative access related complications.

\section{Methodology}

From May 2015 till May 2018, we treated 58 patients included in this study with history of critical limb ischemia as defined by Rutherford classification; rest pain (Category 4), ischemic ulceration not exceeding ulcer of the digits of the foot (Category 5) \& severe ischemic ulcers or frank gangrene (Rutherford category 6). All patients had high risk for anesethia ASA 4, and all patients had intact femoral pulsation with patent iliac arteries. Radiologically, all patients had TASC II D femoropopliteal arterial lesions detected by duplex or CT angiography (Chronic total occlusions of SFA $>20 \mathrm{~cm}$ ) or popliteal artery occlusion. Physical examinations, baseline investigations and ABPI measurement were performed to all patients according to our hospital's protocol.

With patients in the supine position, access to the SFA lesion was achieved from the contralateral or ipsilateral femoral artery. After the sheath placement, 5000 units of heparin were administered. In all cases, antegrade recanalization was initially attempted from the retrograde contralateral femoral artery or through an antegrade ipsilateral approach. However, in 8 cases, recanalization proved to be impossible owing to inability to re-enter the true lumen distal to the occlusion. Therefore, a refined medial retrograde popliteal access at the infracondylar plane was adopted.

All procedures were performed with patients receiving local anesthesia. After a preoperative multislice contrast enhanced CT angiography(Figure 1), standard aortogram and bilateral runoff were done using a biplane conventional angiogram with at least two views 60 degrees apart to evaluate the lesion inflow disease (obstruction or stenosis in the iliac arteries), and the runoff (number of patent tibio-peroneal vessels). 


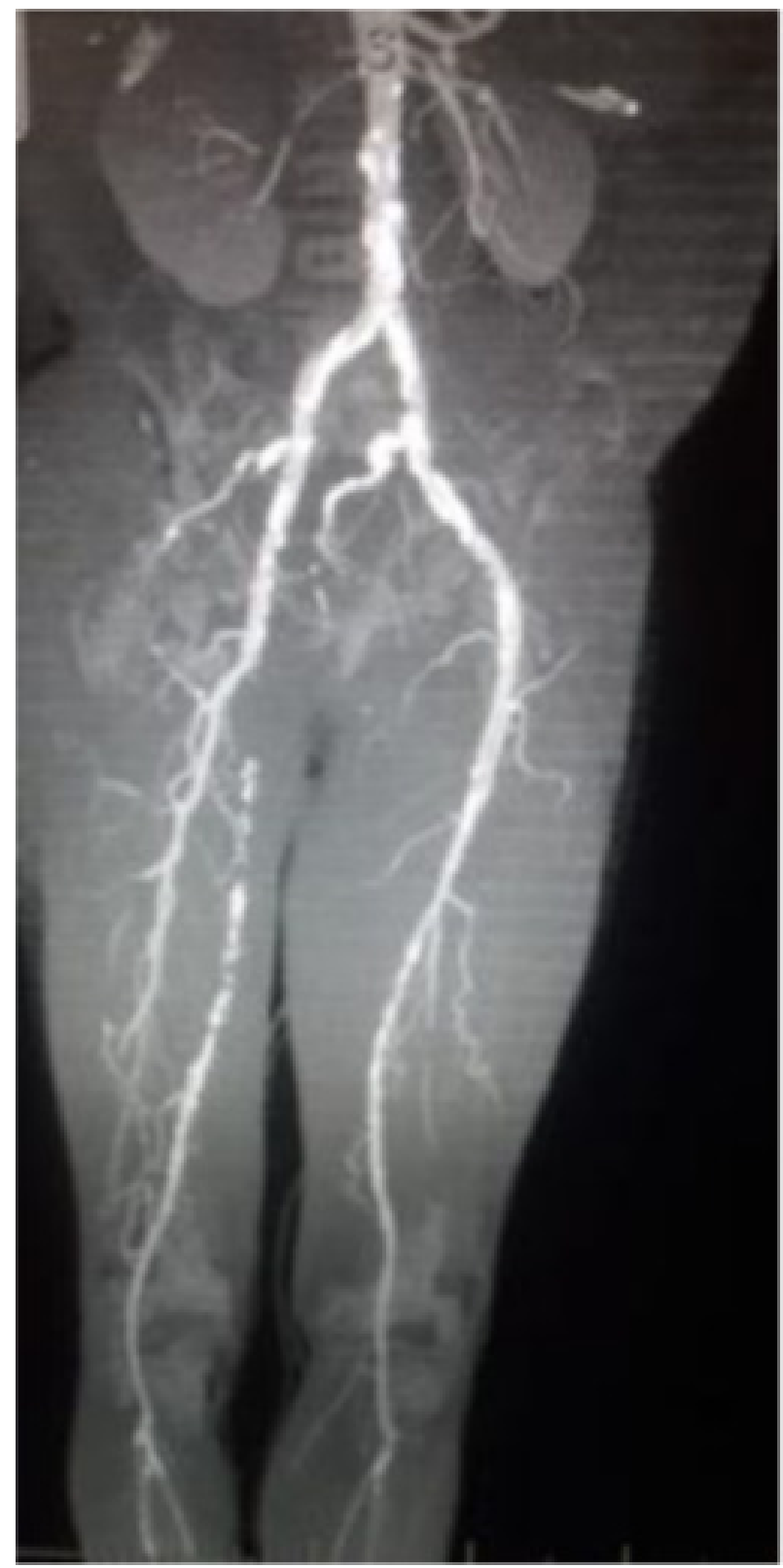

Fig 1. Standard Multislice CT Angiography showing task II-D femoropoplitral lesion.

This was followed by insertion of a standard angle tipped hydrophilic 0.035 guide wire in all cases, stiff hydrophilic 0.035 guide wire was used in $4 / 58$ patients (6.9\%), standard hydrophilic 0.018 in $13 / 58$ patients (22.4\%) and standard hydrophilic 0.014 in 2 patients only (3.4\%). All cases with contralateral CFA retrograde access needed contra catheter 6 French (Cook $\AA$ ) and guiding Rim catheter. 48 patients needed guiding catheter to manipulate the wire to gain its access in the target vessel $(82.75 \%)$ (Bern, Boston Scientific $®$ ), while 10 patients need supporting catheter to increase pushability of GW $(17.2 \%)$ e.g. (Rubicon, Boston Scientific $®)$, (CXI, Cook $($ ) $)$. Sheath size 6 French was used in $(15 / 58$; $25.9 \%)$ and 8 -French in $(43 / 5 ; 74.1 \%)$.

In case of failure of antegrade crossing of the CTO lesion, duplex guided retrograde access was performed using $18 \mathrm{G}$ needle and 0.018 guide wire followed by a 6 French sheath introduction through the popliteal artery via a medial approach. Snaring of the guidewire is then performed using the antegrade sheath, then the antegrade wire crosses the lesion and the procedure is completed (Figures 2,3). For achieving post-procedural hemostasis, direct manual compression of the puncture site was performed for 15 minutes followed by direct angiography. In case of further extravasation, prolonged low-pressure balloon inflation was performed. Complete hemostasis was achieved in all cases.

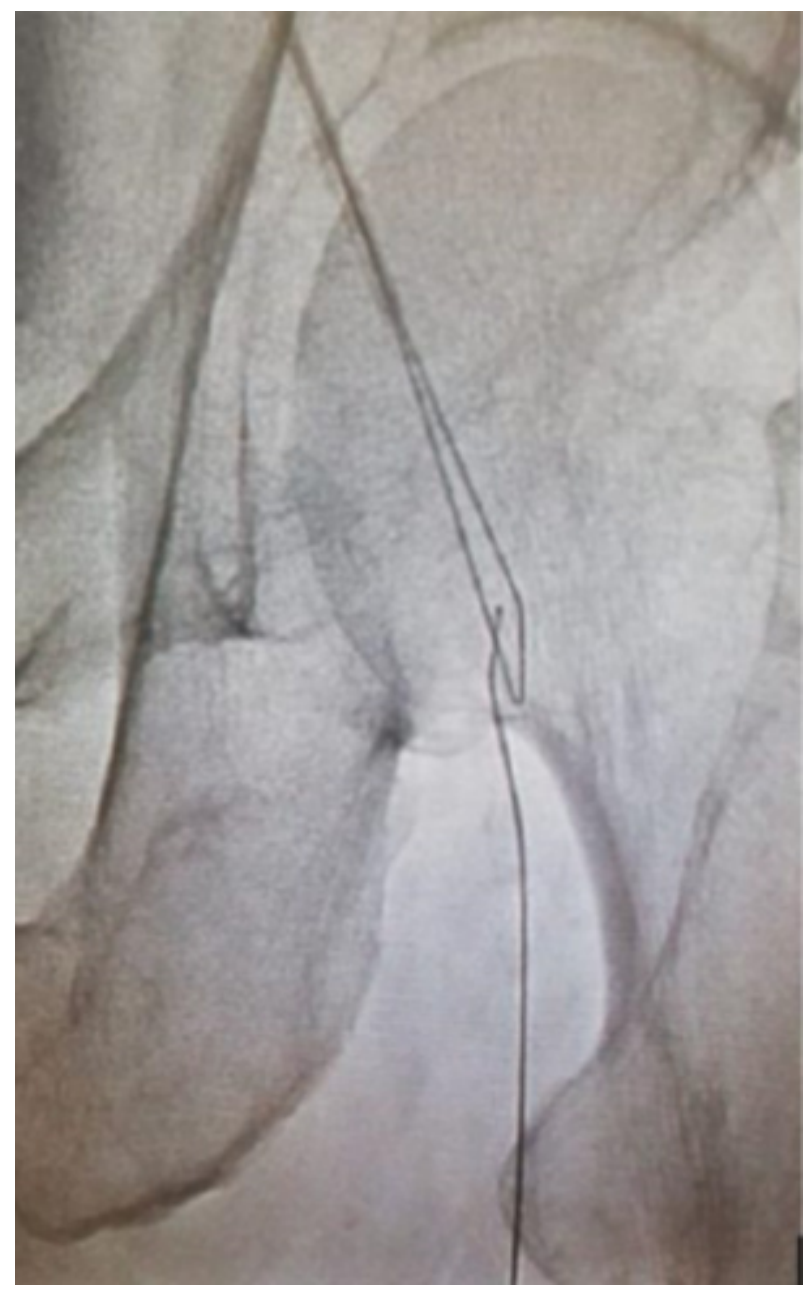

Fig 2. Sharing of retrograde wire throgh the antegrade sheath. 


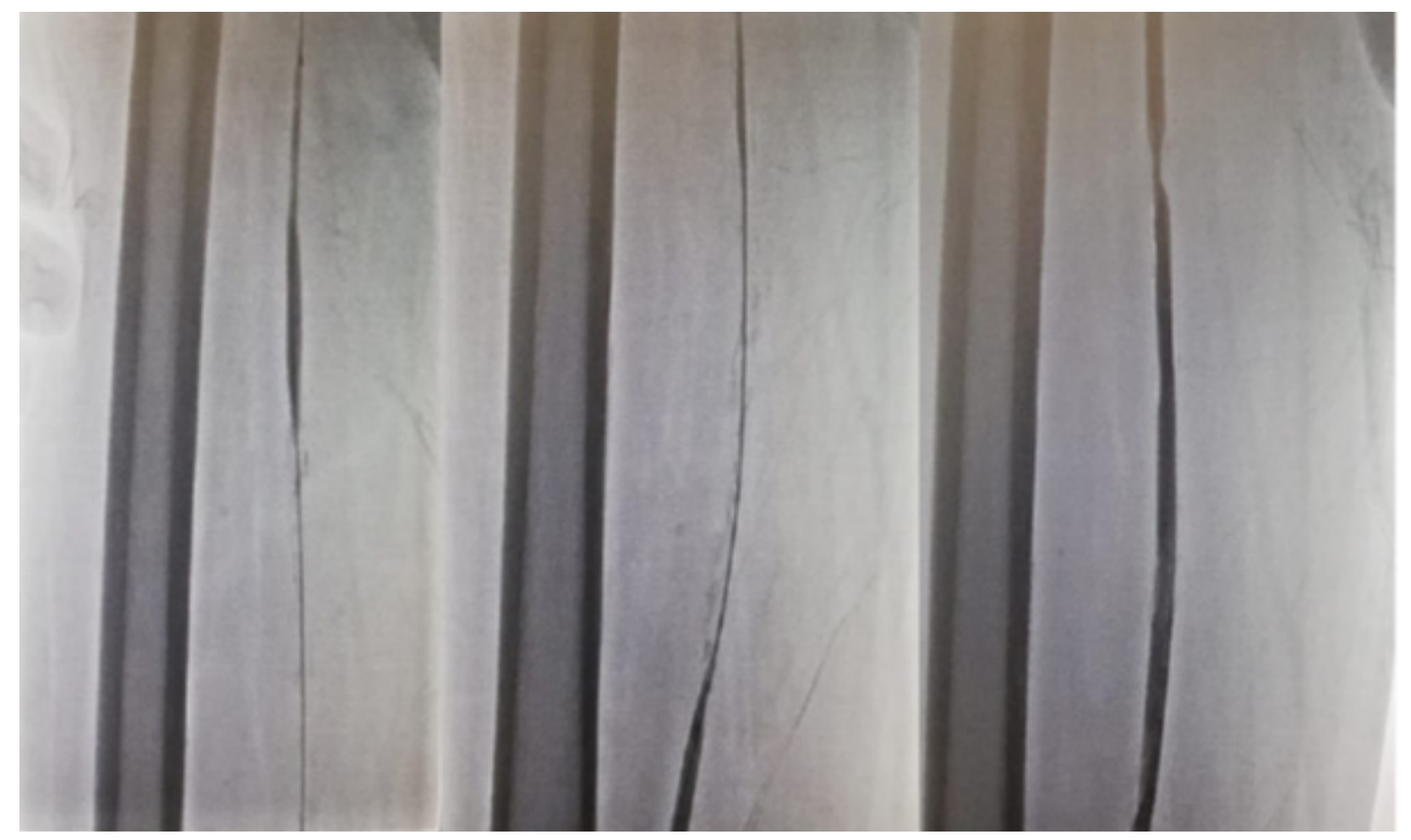

Fig 3. Long $6 \times 300 \mathrm{~mm}$ ballon dilatitation of the SFA.

\section{Statistical Analysis}

Statistical analysis was conducted using SPSS X7 (SPSS, IBM, Chicago, IL, USA). P-values was considered significant if $<0.05$. The continuous variables were presented in the form of mean and standard deviation. The categorical variables were presented as percentages. The demographic data and the comorbidities of the patients were related to the number of patients, whereas the patency data were calculated according to the number of limbs. Chi square tests were used to explore the association between different categorical variables and access groups, ANOVA was used to compare means of non-categorical variables among access site groups, and paired t-test was used to compare mean values of ABPI pre and post intervention.

\section{Results}

58 patients were included: 15 men $(25.9 \%)$ and 43 women $(74.1 \%)$ range of age is between $35-87$ years with mean of 62 (SD; 10.226). Cardiovascular risk factors included Smoking (32/58; 55.2\%) the duration of smoking was between 5 to 50 years with mean of 21.47 (SD;11.02), Hypertension $(41 / 54 ; 75.9 \%)$, obesity $(16 / 58 ; 27.6)$, Diabetes Mellitus (50/58; 86.2\%) in which Insulin dependent DM (25/50; 50\%), hypertension (45/58; 77.6\%), dyslipidemia $(26 / 58 ; 44.8 \%)$, and coronary heart disease $(27 / 58 ; 46.6 \%)$, cerebrovascular accident $(4 / 58 ; 6.9 \%)$, and renal impairment $(1 / 58 ; 1.7 \%)$ (Table 1). 
Table 1: Association and comparing means of patients' demography and pre-existing medical condition with different access group

\begin{tabular}{|c|c|c|c|c|c|}
\hline & & \multicolumn{4}{|c|}{ Access Site } \\
\hline & & $\begin{array}{l}\text { Ispilateral } \\
\text { antegrade }\end{array}$ & $\begin{array}{l}\text { Contralateral } \\
\text { retrograde }\end{array}$ & $\begin{array}{l}\text { Ispilateral } \\
\text { retrograde }\end{array}$ & P-value \\
\hline \multirow{4}{*}{ Gender } & \multirow{2}{*}{ Famale } & 4 & 31 & 8 & \multirow{4}{*}{$0.34 *$} \\
\hline & & $57.1 \%$ & $73.8 \%$ & $88.9 \%$ & \\
\hline & \multirow{2}{*}{ Male } & 3 & 11 & 1 & \\
\hline & & $42.9 \%$ & $26.2 \%$ & $11.1 \%$ & \\
\hline \multirow{4}{*}{ Smoking } & \multirow{2}{*}{ No } & 4 & 16 & 6 & \multirow{4}{*}{$0.23 *$} \\
\hline & & $57.1 \%$ & $38.1 \%$ & $66.7 \%$ & \\
\hline & \multirow{2}{*}{ Yes } & 3 & 26 & 3 & \\
\hline & & $42.9 \%$ & $61.9 \%$ & $33.3 \%$ & \\
\hline \multirow{4}{*}{ Obesity } & \multirow{2}{*}{ No } & 5 & 32 & 5 & \multirow{4}{*}{$0.48^{*}$} \\
\hline & & $71.4 \%$ & $76.2 \%$ & $55.6 \%$ & \\
\hline & \multirow{2}{*}{ Yes } & 2 & 10 & 4 & \\
\hline & & $28.6 \%$ & $23.8 \%$ & $44.4 \%$ & \\
\hline \multirow{4}{*}{ DM } & \multirow{2}{*}{ No } & 1 & 5 & 2 & \multirow{4}{*}{$0.74 \%$} \\
\hline & & $14.3 \%$ & $11.9 \%$ & $22.2 \%$ & \\
\hline & \multirow{2}{*}{ Yes } & 6 & 37 & 7 & \\
\hline & & $85.7 \%$ & $88.1 \%$ & $77.8 \%$ & \\
\hline \multirow{4}{*}{ Type of DM } & \multirow{2}{*}{ IDDM } & 3 & 21 & 1 & \multirow{4}{*}{$0.04 *$} \\
\hline & & $50.0 \%$ & $55.3 \%$ & $11.1 \%$ & \\
\hline & \multirow{2}{*}{ NIDDM } & 3 & 17 & 8 & \\
\hline & & $50.0 \%$ & $44.7 \%$ & $88.9 \%$ & \\
\hline \multirow{4}{*}{ HTN } & \multirow{2}{*}{ No } & 1 & 12 & 0 & \\
\hline & & $14.3 \%$ & $28.6 \%$ & $0.0 \%$ & $006 *$ \\
\hline & Yoc & 6 & 30 & 9 & $0.06^{\uparrow}$ \\
\hline & Yes & $85.7 \%$ & $71.4 \%$ & $100.0 \%$ & \\
\hline & $\mathrm{No}$ & 4 & 23 & 5 & \\
\hline Dudinidamin & NO & $57.1 \%$ & $54.8 \%$ & $55.6 \%$ & \\
\hline Dyslipidemia & Yec & 3 & 19 & 4 & $0.99 *$ \\
\hline & Tes & $42.9 \%$ & $45.2 \%$ & $44.4 \%$ & \\
\hline & $\mathrm{No}$ & 4 & 25 & 2 & \\
\hline Ischemic heart & NO & $57.1 \%$ & $59.5 \%$ & $22.2 \%$ & $011 *$ \\
\hline Disease & $V_{4}$ & 3 & 17 & 7 & $0.11^{\uparrow}$ \\
\hline & res & $42.9 \%$ & $40.5 \%$ & $77.8 \%$ & \\
\hline & $\mathrm{N}$ & 6 & 40 & 9 & \\
\hline Congestive heart & & $85.7 \%$ & $95.2 \%$ & $100.0 \%$ & \\
\hline failure & Yoc & 1 & 2 & 0 & $0.41^{*}$ \\
\hline & res & $14.3 \%$ & $4.8 \%$ & $0.0 \%$ & \\
\hline & $\mathrm{No}$ & 7 & 39 & 8 & \\
\hline Cerebrovascular & & $100.0 \%$ & $92.9 \%$ & $88.9 \%$ & $051 *$ \\
\hline insufficiency & $\mathrm{V}$ & 0 & 3 & 1 & $0.54^{\pi}$ \\
\hline & res & $0.0 \%$ & $7.1 \%$ & $11.1 \%$ & \\
\hline & $\mathrm{N}$ & 6 & 42 & 9 & \\
\hline Dannd & NO & $85.7 \%$ & $100.0 \%$ & $100.0 \%$ & \\
\hline Kendimparminta & Yec & 1 & 0 & 0 & $0.11^{*}$ \\
\hline & res & $14.3 \%$ & $0.0 \%$ & $0.0 \%$ & \\
\hline
\end{tabular}




\begin{tabular}{|c|c|c|c|c|c|}
\hline & & Mean & Std. Deviation & F Value & P-value \\
\hline \multirow{3}{*}{ Age } & $\begin{array}{l}\text { Ispilateral } \\
\text { antegrade }\end{array}$ & 74.00 & 8.446 & \multirow{3}{*}{7.196} & \multirow{3}{*}{$0.002 * *$} \\
\hline & $\begin{array}{l}\text { Contralateral } \\
\text { retrograde }\end{array}$ & 59.71 & 9.689 & & \\
\hline & $\begin{array}{l}\text { Ispilateral } \\
\text { retrograde }\end{array}$ & 63.00 & 7.483 & & \\
\hline \multirow{3}{*}{ Cigaretts per day } & $\begin{array}{l}\text { Ispilateral } \\
\text { antegrade }\end{array}$ & 17.50 & 5.000 & \multirow{3}{*}{1.114} & \multirow{3}{*}{$0.34 * *$} \\
\hline & $\begin{array}{l}\text { Contralateral } \\
\text { retrograde }\end{array}$ & 24.46 & 10.483 & & \\
\hline & $\begin{array}{l}\text { Ispilateral } \\
\text { retrograde }\end{array}$ & 27.50 & 9.574 & & \\
\hline \multirow{3}{*}{$\begin{array}{l}\text { During of smoking } \\
\text { in years }\end{array}$} & $\begin{array}{l}\text { Ispilateral } \\
\text { antegrade }\end{array}$ & 27.50 & 8.660 & \multirow{3}{*}{1.278} & \multirow{3}{*}{$0.29 * *$} \\
\hline & $\begin{array}{l}\text { Contralateral } \\
\text { retrograde }\end{array}$ & 20.46 & 11.504 & & \\
\hline & $\begin{array}{l}\text { Ispilateral } \\
\text { retrograde }\end{array}$ & 26.67 & 10.328 & & \\
\hline $\begin{array}{l}\text { Time of major } \\
\text { amputation in days }\end{array}$ & $\begin{array}{l}\text { Ispilateral } \\
\text { antegrade }\end{array}$ & 8.00 & 0 & 0.552 & $0.59 * *$ \\
\hline
\end{tabular}

*P-value calculated using Likelihood ratio.

**P-value calculated using ANOVA.

We detected $55 / 58$ patients with intact distal run off $(94.8 \%)$, while $3 / 58$ patients with absent distal run off (3.4\%). The lesion site was chronic total occlusion of the Superficial Femoral artery $(48 / 58 ; 82.8 \%)$, popliteal artery $(2 / 58 ; 3.4 \%)$, or both $(4 / 58 ; 6.9 \%)$.
Length of lesion was $20.7+/-10 \mathrm{~cm}$ (SD; 3.457). The duration of procedure was between 30 to 210 Min with mean 108.79 Min (SD; 41.93). (Tables 3,4). 
Table 3: Association and comparing means value of lesion characterristics among different ccess group

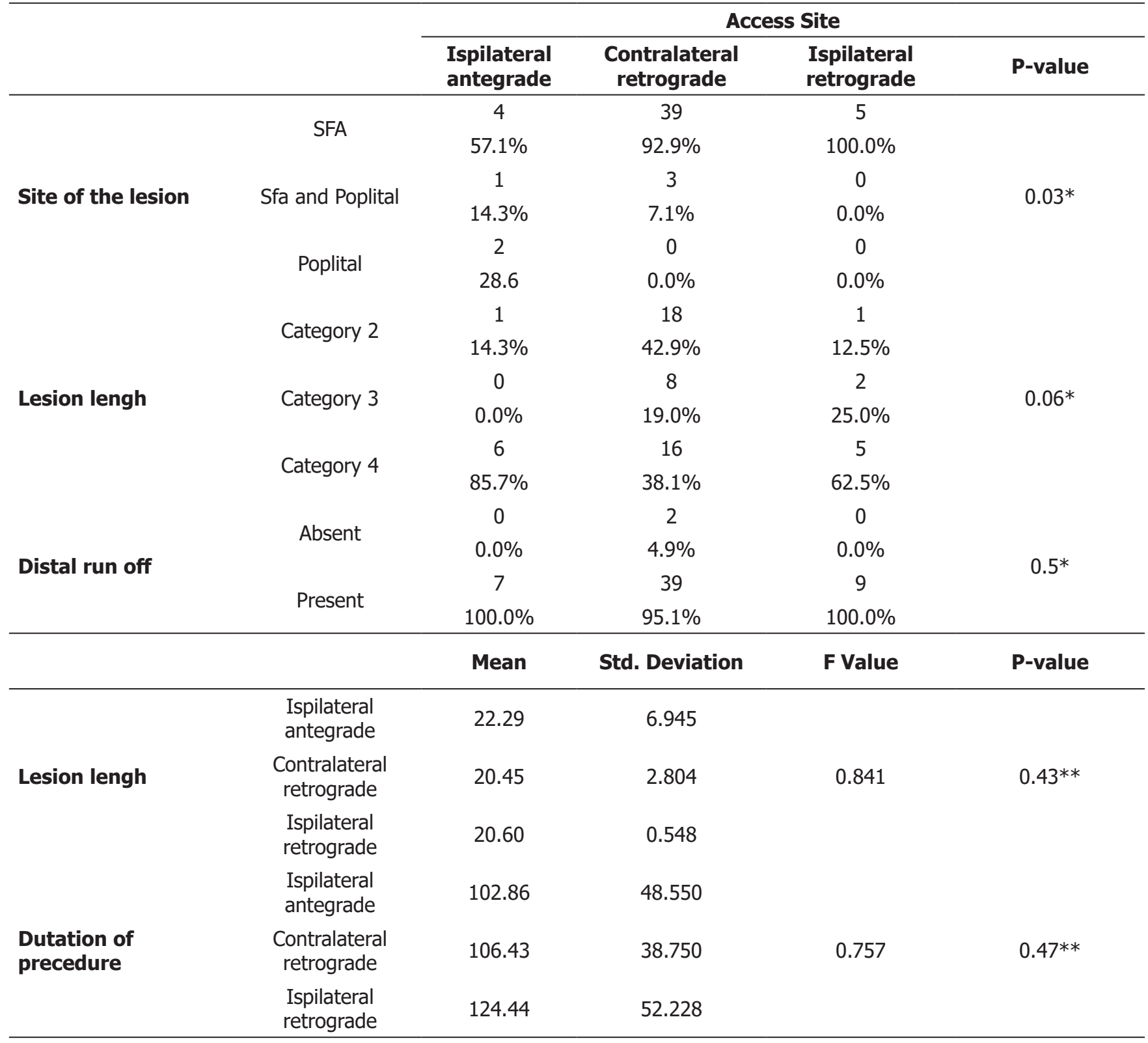




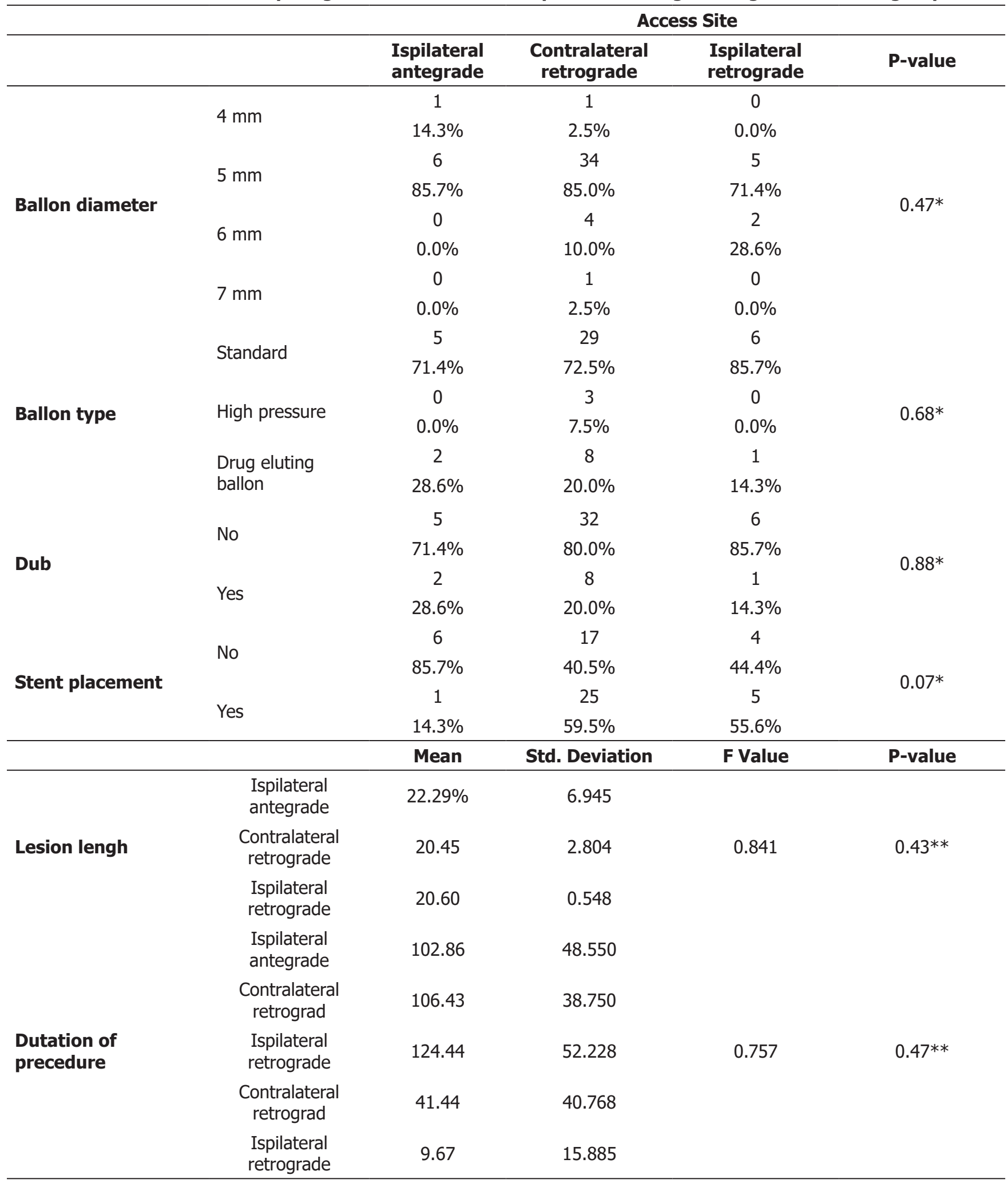

Interventions were performed percutaneously via the Common Femoral Artery (CFA) by an antegrade access $(7 / 58 ; 12.1 \%)$ or an over the-bifurcation approach contralateral CFA access $(42 / 58 ; 72.4 \%)$ or ipsilateral retrograde through popliteal artery access (9/58; 15.5\%). Technical success was highest with contralateral retrograde entailing 40 cases $(95.2 \%)$, followed by ipsilateral ante-grade access ( 6 cases $-85.7 \%$ ) and lowest with ipsilateral retrograde (7 cases-77.8\%) (Figure 4, Table 5). 


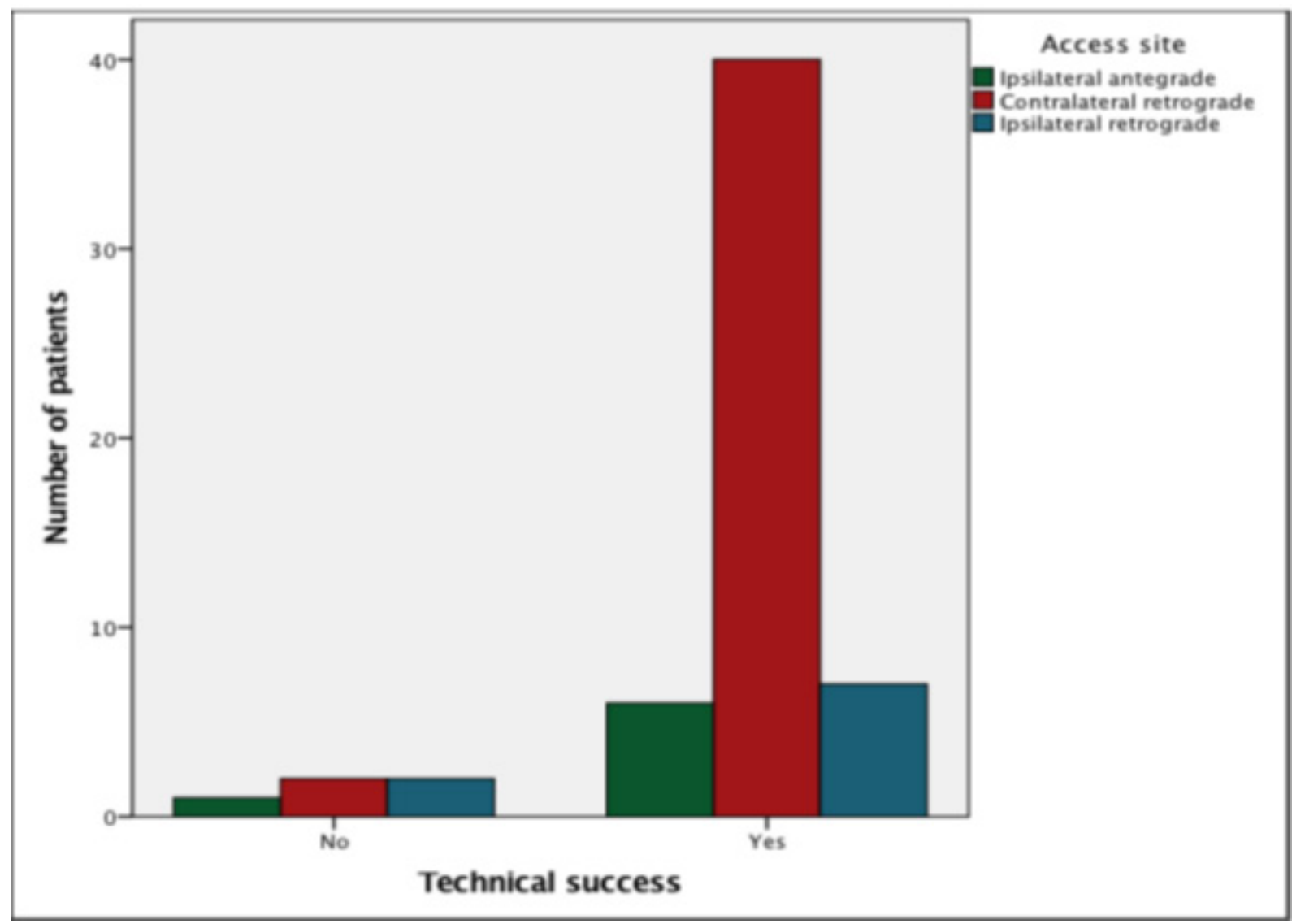

Fig 4: Bar chart showing technical sucess among differnt access groups. 


\begin{tabular}{|c|c|c|c|c|c|}
\hline & & \multicolumn{4}{|c|}{ Access Site } \\
\hline & & $\begin{array}{l}\text { Ispilateral } \\
\text { antegrade }\end{array}$ & $\begin{array}{c}\text { Contralateral } \\
\text { retrograde }\end{array}$ & $\begin{array}{l}\text { Ispilateral } \\
\text { retrograde }\end{array}$ & P-value \\
\hline \multirow{4}{*}{$\begin{array}{l}\text { Procedure related } \\
\text { adverse events }\end{array}$} & \multirow{2}{*}{ No } & 7 & 40 & 9 & \multirow{4}{*}{$0.51^{*}$} \\
\hline & & $100.0 \%$ & $95.2 \%$ & $100.0 \%$ & \\
\hline & \multirow{2}{*}{ Yes } & 0 & 2 & 0 & \\
\hline & & $0.0 \%$ & $4.8 \%$ & $0.0 \%$ & \\
\hline \multirow{4}{*}{ Technical success } & \multirow{2}{*}{ No } & 1 & 2 & 2 & \\
\hline & & $14.3 \%$ & $4.8 \%$ & $22.2 \%$ & \\
\hline & \multirow{2}{*}{ Yes } & 6 & 40 & 7 & \\
\hline & & $85.7 \%$ & $95.2 \%$ & $77.8 \%$ & \\
\hline \multirow{8}{*}{$\begin{array}{l}\text { Postoperative } \\
\text { Rutherford } \\
\text { Classification }\end{array}$} & \multirow{2}{*}{ Asymptomatic } & 0 & 3 & 0 & \multirow{8}{*}{$0.19 *$} \\
\hline & & $0.0 \%$ & $7.1 \%$ & $0.0 \%$ & \\
\hline & \multirow{2}{*}{ Mild claudication } & 6 & 35 & 7 & \\
\hline & & $85.7 \%$ & $83.3 \%$ & $77.8 \%$ & \\
\hline & \multirow{2}{*}{ Iscemic ucler } & 1 & 0 & 0 & \\
\hline & & $14.3 \%$ & $0.0 \%$ & $0.0 \%$ & \\
\hline & \multirow{2}{*}{ Gangrene } & 0 & 4 & 2 & \\
\hline & & $0.0 \%$ & $9.5 \%$ & $22.2 \%$ & \\
\hline \multirow{4}{*}{$\begin{array}{l}\text { Sustain Clinical } \\
\text { Improvement }\end{array}$} & \multirow{2}{*}{ NO } & 0 & 5 & 1 & \multirow{4}{*}{ 0054* } \\
\hline & & $0.0 \%$ & $12.2 \%$ & $12.5 \%$ & \\
\hline & \multirow{2}{*}{ Yes } & 5 & 36 & 7 & \\
\hline & & $100.0 \%$ & $71.8 \%$ & $87.5 \%$ & \\
\hline \multirow{4}{*}{$\begin{array}{l}\text { Binary Restenosis } \\
\text { BS }\end{array}$} & \multirow{2}{*}{ No } & 5 & 28 & 7 & \multirow{4}{*}{$0.15^{*}$} \\
\hline & & $100.0 \%$ & $71.8 \%$ & $87.5 \%$ & \\
\hline & \multirow{2}{*}{ Yes } & 0 & 11 & 1 & \\
\hline & & $0.0 \%$ & $28.2 \%$ & $12.5 \%$ & \\
\hline \multirow{4}{*}{$\begin{array}{l}\text { Target Lesion } \\
\text { Revascularization } \\
\text { TLR }\end{array}$} & $\mathrm{No}$ & 5 & 25 & 6 & \\
\hline & NO & $83.3 \%$ & $61.0 \%$ & $66.7 \%$ & \\
\hline & Yes & 1 & 16 & 3 & $0.53^{\top}$ \\
\hline & & $16.7 \%$ & $39.0 \%$ & $33.3 \%$ & \\
\hline Target Extremity & & 6 & 41 & 9 & \\
\hline $\begin{array}{l}\text { Revascularization } \\
\text { TLR }\end{array}$ & No & $100.0 \%$ & $100.0 \%$ & $100.0 \%$ & - \\
\hline & $\mathrm{No}$ & 1 & 3 & 2 & \\
\hline $\begin{array}{l}\text { Immediate } \\
\text { Haemodvnamic }\end{array}$ & INO & $16.7 \%$ & $7.3 \%$ & $22.2 \%$ & 24 \\
\hline $\begin{array}{l}\text { Haemoaynamic } \\
\text { Improvement }\end{array}$ & Yes & 5 & 38 & 7 & $0.42^{\top}$ \\
\hline & Tes & $83.3 \%$ & $92.7 \%$ & $77.8 \%$ & \\
\hline & & 1 & 7 & 2 & \\
\hline Sustai & NO & $16.7 \%$ & $17.1 \%$ & $22.2 \%$ & ० १४* \\
\hline Improvement & Yes & 5 & 34 & 7 & 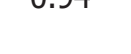 \\
\hline & Tes & $83.3 \%$ & $82.9 \%$ & $77.8 \%$ & \\
\hline & No & 7 & 40 & 9 & \\
\hline Maior Ampulation & IVO & $100.0 \%$ & $97.6 \%$ & $100.0 \%$ & \\
\hline Major Ampuldtion & Yes & 0 & 1 & 0 & $0 . / L^{\top}$ \\
\hline & S & $0.0 \%$ & $2.4 \%$ & $0.0 \%$ & \\
\hline
\end{tabular}




\begin{tabular}{|c|c|c|c|c|c|}
\hline \multirow{4}{*}{ Limb Salvage } & \multirow{2}{*}{ No } & 0 & 1 & 0 & \multirow{4}{*}{$0.72 *$} \\
\hline & & $0.0 \%$ & $2.4 \%$ & $0.0 \%$ & \\
\hline & \multirow{2}{*}{ Yes } & 7 & 40 & 9 & \\
\hline & & $100.0 \%$ & $97.6 \%$ & $100.0 \%$ & \\
\hline & & Mean & Std. Deviation & F Value & P-value \\
\hline \multirow{3}{*}{$\begin{array}{l}\text { Post-operative Right } \\
\text { ABPI }\end{array}$} & $\begin{array}{l}\text { Ispilateral } \\
\text { antegrade }\end{array}$ & 0.6671 & 0.07088 & \multirow{3}{*}{0.058} & \multirow{3}{*}{$0.94 * *$} \\
\hline & $\begin{array}{l}\text { Contralateral } \\
\text { retrograde }\end{array}$ & 0.6902 & 0.25156 & & \\
\hline & $\begin{array}{l}\text { Ispilateral } \\
\text { retrograde }\end{array}$ & 0.7088 & 0.23703 & & \\
\hline \multirow{3}{*}{$\begin{array}{l}\text { Post-operative Left } \\
\text { ABPI }\end{array}$} & $\begin{array}{l}\text { Ispilateral } \\
\text { antegrade }\end{array}$ & 0.7143 & 0.13138 & \multirow{3}{*}{0.252} & \multirow{3}{*}{$0.77 * *$} \\
\hline & $\begin{array}{l}\text { Contralateral } \\
\text { retrograde }\end{array}$ & 0.7283 & 0.17452 & & \\
\hline & $\begin{array}{l}\text { Ispilateral } \\
\text { retrograde }\end{array}$ & 0.7750 & 0.12550 & & \\
\hline \multirow{3}{*}{$\begin{array}{l}\text { Time to } \\
\text { Target Lesion } \\
\text { Revascularization } \\
\text { weeks }\end{array}$} & $\begin{array}{l}\text { Ispilateral } \\
\text { antegrade }\end{array}$ & 1.00 & 0 & \multirow{3}{*}{1.251} & \multirow{3}{*}{$0.31 * *$} \\
\hline & $\begin{array}{l}\text { Contralateral } \\
\text { retrograde }\end{array}$ & 41.44 & 40.768 & & \\
\hline & $\begin{array}{l}\text { Ispilateral } \\
\text { retrograde }\end{array}$ & 9.67 & 15.885 & & \\
\hline
\end{tabular}

* P-value calculated using Likeihood ratio

** P-value calculated using ANOVA

Mean pre-intervention ABPI was 0.54 (SD 0.19) and 0.57 (SD 0.22), and the post-intervention ABPI was 0.69 (SD 0.23) and 0.73 (SD 0.16) for the right and left limbs respectively, which was statistically significant with a p-value of $<0.0001$ (Figure 5, Table 6).

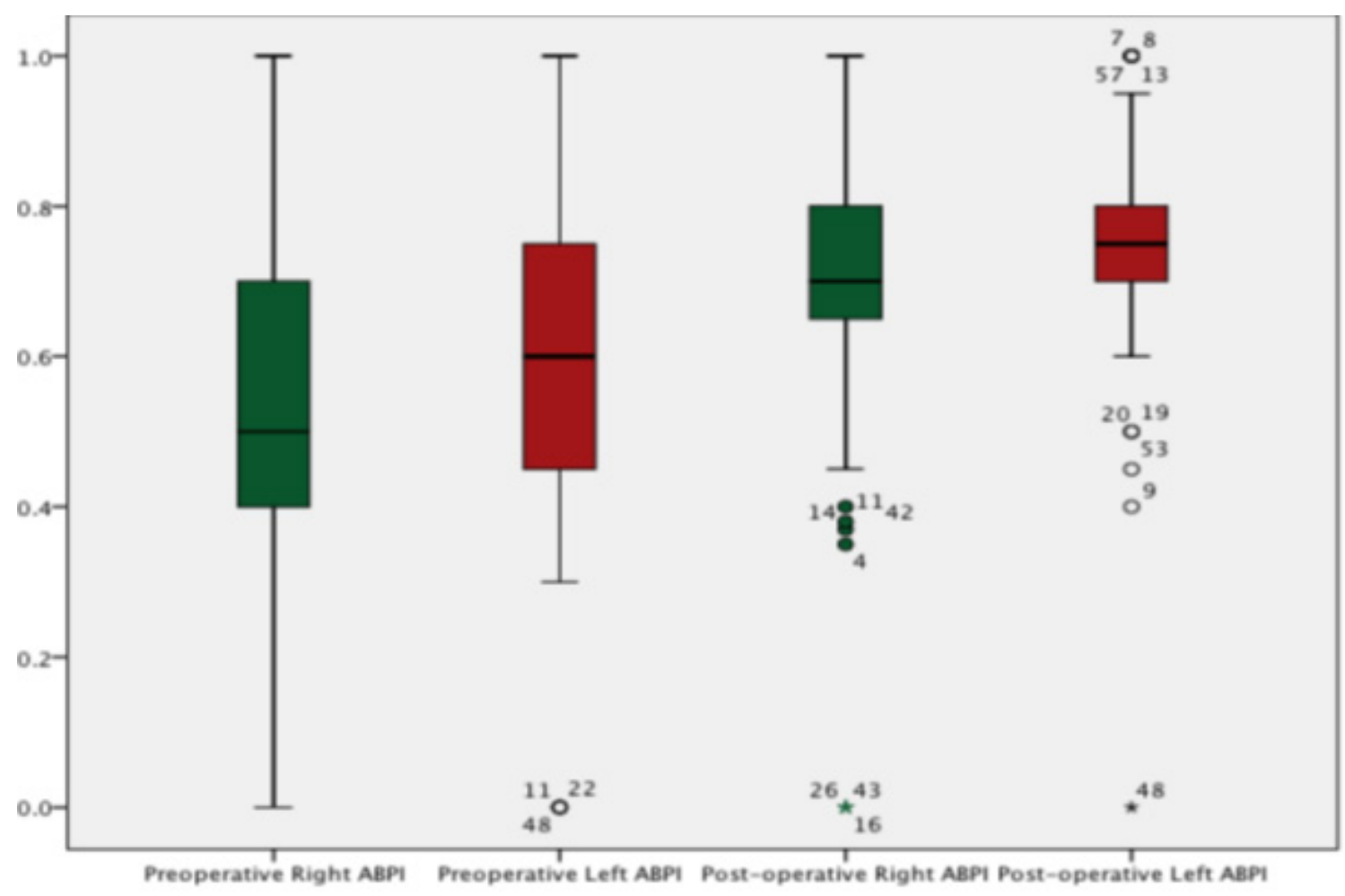

Fig 5: Boxplot representation of both pre intervention ABPI measurement. 
Table 6: Comparing mean values of pre-intervention and post-intervention ABPIs

\begin{tabular}{lccc}
\hline & Mean & Std. Deviation & P-value \\
\hline Pre-operative Right ABPI & 0.54 & 0.19 & $<0.0001$ \\
Post-operative Right ABPI & 0.69 & 0.23 & $<0.0001$ \\
Pre-operative Left ABPI & 0.57 & 0.22 & 0.16 \\
Post-operative Left ABPI & 0.73 & 0.16 & \\
\hline
\end{tabular}

* P-value calculated using paired sample t-test

\section{Discussion}

Endovascular treatment for chronic total occlusion of the femoro-popliteal artery is usually managed from an antegrade ipsilateral common femoral artery (CFA) approach or via retrograde contralateral CFA. Manipulation from the contralateral CFA may become difficult in patients with tortuous iliac arteries or an aorto-bifemoral Y-prosthesis used to treat a previous abdominal aortic aneurysm. Compared to the contralateral retrograde approach, the ipsilateral antegrade provides better pushability. However, there are a plethora of circumstances in which an ipsilateral CFA access would be difficult or contraindicated, such as a flush SFA occlusion without proximal stump, obesity, surgical scars in the groin, and lesions involving the CFA. When antegrade recanalization fails, a retrograde transpopliteal approach can be used as an alternative.

The retrograde popliteal approach requires that the patient be turned to a prone position. However, with the patient prone, the maneuvers from the femoral access are difficult for the interventionist and often prolong the procedure. Furthermore, patients in the prone position are apt to feel fatigue, especially those who are obese or have impaired respiratory function. ${ }^{11}$ In addition, a subintimal revascularization with a planned combined subintimal arterial flossing with antegrade-retrograde intervention (SAFARI) can be adopted to improve the success rate and avoid damage to important collaterals. ${ }^{12}$

\section{Conclusion}

Trans-popliteal retrograde approach can be accomplished safely and efficiently using a medial infracondylar retrograde popliteal puncture, with the patient in the supine position. Furthermore, this retrograde popliteal approach can be considered a valid alternative for Superficial Femoral Artery, or proximal popliteal recanalization after a failed antegrade approach.

\section{References}

1. Peach G, Griffin M, Jones KG, Thompson MM, Hinchcliffe RJ: Diagnosis and management of peripheral arterial disease. BMJ. 2012; 345: 520-528.
2. McDermott $M$, Greenland $P$, Liu $K$, Guralnik J, Criqui $\mathrm{M}$, Dolan $\mathrm{N}$ et al: Leg symptoms in peripheral arterial disease: associated clinical characteristics and functional impairment. JAMA. 2001; 286(13): 1599-606.

3. Hiatt WR, Fowkes FG, Heizer G, Berger JS, Baumgartner I, Held P, Katona BG, Mahaffey KW, Norgren L, Jones WS, Blomster J, Millegard M, Reist C, Patel MR: Ticagrelor versus clopidogrel in symptomatic peripheral artery disease. $N$ Engl J Med. 2017; 376: 32-40.

4. Norgren L, Pande RL, Preux PM, Stoffers HE: Treat-Jacobson D. Measurement and interpretation of the ankle-brachial index: $\mathrm{A}$ scientific statement from the American Heart Association. Circulation. 2012; 126: 28902909.

5. Bosiers M, Peeters P, Tessarek J, Deloose K, Strickler S: The Zilver PTX Single-Arm Study Investigators. The Zilver_ PTX_ Single Arm Study: 12-month results from the TASC C/D lesion subgroup. J Cardiovasc Surg (Torino). 2013; 54: 115-122.

6. Adam DJ, Beard JD, Cleveland T, Bell J, Bradbury AW, Forbes JF: Bypass versus angioplasty in severe ischaemia of the leg (BASIL): Multicenter, randomized controlled trial. Lancet 2005; 366: 1925-1934.

7. Bradbury AW, Adam DJ, Bell J, Forbes JF, Fowkes FGR, Gillespie I, et al: Bypass versus Angioplasty in Severe Ischaemia of the Leg (BASIL) trial: an intention-to-treat analysis of amputation-free and overall survival in patients randomized to a bypass surgery-first or a balloon angioplastyfirst revascularization strategy. J Vasc Surg. 2010; 51(Suppl 10): 5S-17S.

8. Tonnesen $\mathrm{KH}$, Sager $\mathrm{P}$, Karle $\mathrm{A}$, Henrisen $\mathrm{L}$, Jorgensen $\mathrm{B}$ : Percutaneous transluminal angioplasty of the superficial femoral artery by retrograde catheterization via the popliteal artery. Cardiovasc Intervent Radiol. 1988; 11: 127-31. 
9. Silvestro $M$, Palena LM, Manzi M, GómezJabalera E, Vishwanath D, Casini A, Ferraresi $\mathrm{R}$ : Anterolateral retrograde access to the distal popliteal artery and to the tibioperoneal trunk for recanalization of femoro-popliteal chronic total occlusions. Journal of Vascular Surgery. https://doi.org/10.1016/j.jvs. 2018; 05: 231.

10. Komshian S, Cheng TW, Farber A, Schermerhorn ML, Kalish JA, Rybin D: Vascular Quality Initiative. (2018). Retrograde popliteal access to treat femoro-popliteal artery occlusive disease.
Journal
of
Vascular
Surgery. 2018;

68(1): 161-167. https://doi.org/10.1016/j.jvs. 2017; 12: 022.

11. Spinosa DJ, Harthun NL, Bissonette EA, Cage $\mathrm{D}$, Leung DA, Angle JF, Matsumoto, $\mathrm{AH}$ : Subintimal arterial flossing with antegraderetrograde intervention (SAFARI) for subintimal recanalization to treat chronic critical limb ischemia. Journal of Vascular and Interventional Radiology. 2005; 16(1): 37-44. https://doi. org/10.1097/01.RVI.0000141336.53745.4A. 